\title{
Hybrid iterative algorithms for the split common fixed point problems
}

\author{
Jong Soo Jung \\ Department of Mathematics, Dong-A University, Busan 49315, Korea.
}

Communicated by Y. J. Cho

\begin{abstract}
In this paper, we introduce two iterative algorithms (one implicit algorithm and one explicit algorithm) based on the hybrid steepest descent method for solving the split common fixed point problems. We establish the strong convergence of the sequences generated by the proposed algorithms to a solution of the split common fixed point problems, which is also a solution of a certain variational inequality. In particular, the minimum norm solution of the split common fixed point problems is obtained. As applications, variational problems and equilibrium problems are considered. (C)2017 All rights reserved.
\end{abstract}

Keywords: Split common fixed point problem, firmly nonexpansive mapping, nonexpansive mapping, variational inequality, minimum-norm, $\rho$-Lipschitzian and $\eta$-strongly monotone operator, bounded linear operator, variational problems, equilibrium problems, iterative algorithms.

2010 MSC: 47J05, 49J40, 49J52, 47J20, 47H10.

\section{Introduction}

The split feasibility problem (in short, SFP) is formulated as

$$
\text { find } x^{*} \in C \text { such that } A x^{*} \in Q \text {, }
$$

where $\mathrm{C}$ and $\mathrm{Q}$ are two nonempty closed convex subsets of real Hilbert spaces $\mathrm{H}_{1}$ and $\mathrm{H}_{2}$, respectively, and $A: H_{1} \rightarrow H_{2}$ is a bounded linear operator. The SFP (1.1) in finite-dimensional Hilbert spaces was first introduced by Censor and Elfving [8] for modeling inverse problems which arise in phase retrievals and in medical image reconstruction [4]. In [7, 9, 10], it has been shown that the SPF (1.1) can also be used to model the intensity-modulated radiation therapy. Various iterative algorithms have been studied to solve the SFP (1.1), see, e.g., [8, 14, 16, 18, 22, 25, 27, 30-32] and the references therein. In particular, Jung [16] introduced iterative algorithms based on the Yamada's hybrid steepest descent method [28] for solving SFP (1.1). He established strong convergence of sequences generated by the proposed algorithms to a solution of SFP (1.1), which is a solution of a certain variational inequality defined over the set of solutions of SFP (1.1).

Recently, several split type feasibility problems have been considered because of their applications in science, engineering, medical sciences, etc. One of the split type problems is the split common fixed point

Email address: jungjs@dau.ac.kr (Jong Soo Jung)

doi:10.22436/jnsa.010.04.72 
problem (in short, SCFPP) which is to find a fixed point of an operator such that its image under the bounded linear operator is a fixed point of another operator, that is,

$$
\text { find } x^{*} \in \operatorname{Fix}(T) \text { such that } A x^{*} \in \operatorname{Fix}(S) \text {, }
$$

where $\operatorname{Fix}(T)$ and $\operatorname{Fix}(S)$ denote the set of fixed points of the operators $T: H_{1} \rightarrow H_{1}$ with $\operatorname{Fix}(T) \neq \emptyset$ and $S: \mathrm{H}_{2} \rightarrow \mathrm{H}_{2}$ with $\operatorname{Fix}(\mathrm{S}) \neq \emptyset$, respectively. We denote by $\Omega$ the set of solutions of the SCFPP (1.2) and assume that $\Omega \neq \emptyset$. The SCFPP (1.2) was introduced by Censor and Segal [11]. They considered a parallel algorithm for solving the SCFPP (1.2) for a class of directed operators in finite dimensional spaces. Later, Ansari et al. [2], Cui and Wang [13], Krailkaew and Saejung [17] and Moudafi [20, 21] proposed different kinds of algorithms for solving SCFPP (1.2) in the Hilbert space setting.

In this paper, motivated by the works $[2,16]$, we present two iterative algorithms based on Yamada's the hybrid steepest descent method [28] for solving the SCFPP (1.2). First, we introduce an implicit algorithm. Next, by discretizing the continuous implicit algorithm, we provide an explicit algorithm. Under some appropriate conditions, we show the strong convergence of proposed algorithms to some solution of the SCFPP (1.2) which solves a certain variational inequality. As special cases, we obtain two algorithms which converges strongly to the minimum norm solution of the SCFPP (1.2). As applications, using our iterative algorithms, we study some variational inequality problem and equilibrium problems. The paper can be considered as a continuation of study for solving the SCFPP (1.2) via fixed point methods.

\section{Preliminaries and lemmas}

Let $\mathrm{H}$ be a real Hilbert space with inner product $\langle\cdot, \cdot\rangle$ and norm $\|\cdot\|$, and let $\mathrm{K}$ be a nonempty closed convex subset of $\mathrm{H}$. Recall that the (nearest point or metric) projection from $\mathrm{H}$ onto $\mathrm{K}$, denoted by $\mathrm{P}_{\mathrm{K}}$, is defined in such a way that, for each $x \in H, P_{K} x$ is the unique point in $K$ with the property

$$
\left\|x-P_{K}(x)\right\|=\min \{\|x-y\|: y \in K\} .
$$

We recall $([1,5,6,29])$ that

(1) a mapping $f: H \rightarrow H$ is $k$-contractive if $\|f x-f y\| \leqslant k\|x-y\|$ for a constant $k \in[0,1)$ and $\forall x, y \in H$;

(2) a mapping $\mathrm{V}: \mathrm{H} \rightarrow \mathrm{H}$ is l-Lipschitzian if $\|\mathrm{Vx}-\mathrm{Vy}\| \leqslant l\|x-y\|$ for a constant $l \in[0, \infty)$ and $\forall x, y \in \mathrm{H}$;

(3) a mapping $\mathrm{T}: \mathrm{H} \rightarrow \mathrm{H}$ is nonexpansive if $\|\mathrm{T} x-\mathrm{Ty}\| \leqslant\|x-y\|, \forall x, y \in H$;

(4) a mapping $\mathrm{T}: \mathrm{H} \rightarrow \mathrm{H}$ is strongly nonexpansive if $\mathrm{T}$ is nonexpansive and

$$
\lim _{n \rightarrow \infty}\left\|\left(x_{n}-y_{n}\right)-\left(T x_{n}-T y_{n}\right)\right\|=0,
$$

whenever $\left\{x_{n}\right\}$ and $\left\{y_{n}\right\}$ are bounded sequences in $H$ and $\lim _{n \rightarrow \infty}\left(\left\|x_{n}-y_{n}\right\|-\left\|T x_{n}-T y_{n}\right\|\right)=0$;

(5) a mapping $\mathrm{T}: \mathrm{H} \rightarrow \mathrm{H}$ is firmly quasi-nonexpansive if $\|\mathrm{T} x-p\|^{2} \leqslant\|x-p\|^{2}-\|\mathrm{T} x-p\|^{2}$ for all $x \in \mathrm{H}$ and $p \in \operatorname{Fix}(T)$;

(6) a mapping $T: H \rightarrow H$ is averaged if $T=(1-v) I+v G$, where $v \in(0,1)$ and $G: H \rightarrow H$ is nonexpansive. In this case, we also say that $T$ is $v$-averaged;

(7) a mapping $A: H \rightarrow H$ is monotone if $\langle A x-A y, x-y\rangle \geqslant 0, \forall x, y \in H$;

(8) a mapping $\mathrm{T}: \mathrm{H} \rightarrow \mathrm{H}$ is $\alpha$-inverse strongly monotone $(\alpha$-ism) if there exists $\alpha>0$ such that

$$
\langle\mathrm{T} x-\mathrm{T} y, x-y\rangle \geqslant \alpha\|\mathrm{T} x-\mathrm{T} y\|^{2}, \forall x, y \in H ;
$$

(9) an operator $\mathrm{F}: \mathrm{H} \rightarrow \mathrm{H}$ is $\mathrm{k}$-Lipschitzian and $\eta$-strongly monotone with constants $\mathrm{k}>0$ and $\eta>0$ if $\|F x-F y\| \leqslant k\|x-y\|$ and $\langle F x-F y, x-y\rangle \geqslant \eta\|x-y\|^{2}, \forall x, y \in H$, respectively. 
The following result is well-known.

Proposition 2.1 ([5]). Let $\mathrm{H}$ be a real Hilbert space, and let $\mathrm{T}: \mathrm{H} \rightarrow \mathrm{H}$ be an operator.

(a) If $\mathrm{T}$ is $v$-ism, then for $\gamma>0, \gamma \mathrm{T}$ is $\frac{v}{\gamma}$-ism.

(b) $\mathrm{T}$ is averaged if and only if the complement $\mathrm{I}-\mathrm{T}$ is $\boldsymbol{v}$-ism for some $v>\frac{1}{2}$. Indeed, for $\alpha \in(0,1), \mathrm{T}$ is $\alpha$-averaged if and only if $(\mathrm{I}-\mathrm{T})$ is $\frac{1}{2 \alpha}$-ism.

(c) The composite of finitely many averaged mappings is averaged.

(d) If the mappings $\left\{\mathrm{T}_{i}\right\}_{i=1}^{\mathrm{N}}$ are averaged and have a common fixed point, then

$$
\bigcap_{i=1}^{N} \operatorname{Fix}\left(T_{i}\right)=\operatorname{Fix}\left(T_{1} \cdots T_{N}\right) .
$$

As in [2], using Proposition 2.1, we can prove the following. So we omit its proof.

Proposition 2.2. Let $\mathrm{H}_{1}$ and $\mathrm{H}_{2}$ be real Hilbert spaces, let $\mathrm{A}: \mathrm{H}_{1}-\mathrm{H}_{2}$ be a bounded linear operator, let $\mathrm{A}^{*}$ be the adjoint $\mathrm{A}$, and let $\mathrm{S}: \mathrm{H}_{2} \rightarrow \mathrm{H}_{2}$ be a nonexpansive mapping with $\operatorname{Fix}(\mathrm{S}) \neq \emptyset$. Then

(i) $A^{*}(\mathrm{I}-\mathrm{S}) \mathrm{A}$ is $\frac{1}{2\|\mathrm{~A}\|^{2}}-i s m$;

(ii) $\mathrm{U}:=\mathrm{I}-\gamma \mathrm{A}^{*}(\mathrm{I}-\mathrm{S}) \mathrm{A}$ is averaged for $\gamma \in\left(0, \frac{1}{\|\mathrm{~A}\|^{2}}\right)$ and hence $\mathrm{U}$ is nonexpansive;

(iii) $A x \in \operatorname{Fix}(S)$ implies $x \in \operatorname{Fix}(\mathrm{U})$, and $x \in \operatorname{Fix}(\mathrm{U})$ implies $A x \in \operatorname{Fix}(S)$.

We also need the following lemmas for the proof of our main results.

Lemma 2.3 ([1]). In a real Hilbert space $\mathrm{H}$, the following inequality holds:

$$
\|x+y\|^{2} \leqslant\|x\|^{2}+2\langle y, x+y\rangle, \quad \forall x, y \in H .
$$

Lemma 2.4 (Demiclosedness principle, [15]). Let C be a nonempty closed convex subset of a real Hilbert space $\mathrm{H}$, and let $\mathrm{S}: \mathrm{C} \rightarrow \mathrm{C}$ be a nonexpansive mapping. Then, the mapping $\mathrm{I}-\mathrm{S}$ is demiclosed. That is, if $\left\{\mathrm{x}_{\mathrm{n}}\right\}$ is a sequence in $C$ such that $x_{n} \rightarrow x^{*}$ and $(I-S) x_{n} \rightarrow y$, then $(I-S) x=y$.

Lemma 2.5 ([19]). Let $\mathrm{C}$ be a nonempty closed convex subset of a real Hilbert space $\mathrm{H}$. Assume that the mapping $\mathrm{F}: \mathrm{C} \rightarrow \mathrm{H}$ is monotone and weakly continuous along segments (i.e., $\mathrm{F}(\mathrm{x}+\mathrm{ty}) \rightarrow \mathrm{F}(\mathrm{x})$ as $\mathrm{t} \rightarrow 0$ ). Then the variational inequality

$$
x^{*} \in \mathrm{C}, \quad\left\langle\mathrm{F} x^{*}, x-x^{*}\right\rangle \geqslant 0, \quad x \in C,
$$

is equivalent to the dual variational inequality

$$
x^{*} \in \mathrm{C}, \quad\left\langle\mathrm{F} x, x-x^{*}\right\rangle \geqslant 0, \quad x \in \mathrm{C} .
$$

Lemma 2.6 ([23]). Let $\left\{x_{n}\right\}$ and $\left\{z_{n}\right\}$ be bounded sequences in a Banach space $E$ and $\left\{\gamma_{n}\right\}$ be a sequence in $[0,1]$ which satisfies the following condition:

$$
0<\liminf _{n \rightarrow \infty} \gamma_{n} \leqslant \limsup _{n \rightarrow \infty} \gamma_{n}<1 .
$$

Suppose that $x_{n+1}=\gamma_{n} x_{n}+\left(1-\gamma_{n}\right) z_{n}, n \geqslant 0$, and

$$
\limsup _{n \rightarrow \infty}\left(\left\|z_{n+1}-z_{n}\right\|-\left\|x_{n+1}-x_{n}\right\|\right) \leqslant 0 .
$$

Then $\lim _{n}\left\|z_{n}-x_{n}\right\|=0$.

Lemma 2.7 ([26]). Let $\left\{s_{n}\right\}$ be a sequence of non-negative real numbers satisfying

$$
s_{n+1} \leqslant\left(1-\lambda_{n}\right) s_{n}+\lambda_{n} \delta_{n}, \quad \forall n \geqslant 0,
$$

where $\left\{\lambda_{n}\right\}$ and $\left\{\delta_{n}\right\}$ satisfy the following conditions: 
(i) $\left\{\lambda_{n}\right\} \subset[0,1]$ and $\sum_{n=0}^{\infty} \lambda_{n}=\infty$,

(ii) $\limsup _{n \rightarrow \infty} \delta_{n} \leqslant 0$ or $\sum_{n=0}^{\infty} \lambda_{n}\left|\delta_{n}\right|<\infty$.

Then $\lim _{n \rightarrow \infty} s_{n}=0$.

The following lemma can be easily proven, and therefore, we omit the proof (see also [28]).

Lemma 2.8. Let $\mathrm{H}$ be a real Hilbert space $\mathrm{H}$. Let $\mathrm{F}: \mathrm{H} \rightarrow \mathrm{H}$ be a $\mathrm{k}$-Lipschizian and $\eta$-strongly monotone operator with constants $\mathrm{K}>0$ and $\eta>0$. Let $0<\mu<\frac{2 \eta}{k^{2}}$ and $0<\mathrm{t}<\xi \leqslant 1$. Then $\mathrm{G}:=\xi \mathrm{I}-\mathrm{t} \mu \mathrm{F}: \mathrm{H} \rightarrow \mathrm{H}$ is a contractive mapping with constant $\xi-\mathrm{t} \tau$, where $\tau=1-\sqrt{1-\mu\left(2 \eta-\mu \kappa^{2}\right)}$.

From now, we will use the following notations:

- $x_{n} \rightarrow x$ stands for the weak convergence of $\left\{x_{n}\right\}$ to $x$;

- $x_{n} \rightarrow x$ stands for the strong convergence of $\left\{x_{n}\right\}$ to $x$.

\section{Iterative algorithms}

Throughout the rest of this paper, we always assume the followings:

- $\mathrm{H}_{1}$ and $\mathrm{H}_{2}$ are real Hilbert spaces;

- $A: H_{1} \rightarrow H_{2}$ is a bounded linear operator and $A^{*}$ is the adjoint of $A$;

- $\mathrm{T}: \mathrm{H}_{1} \rightarrow \mathrm{H}_{1}$ is a firmly nonexpansive mapping with $\operatorname{Fix}(\mathrm{T}) \neq \emptyset$;

- $\mathrm{S}: \mathrm{H}_{2} \rightarrow \mathrm{H}_{2}$ is a nonexpansive mapping with $\operatorname{Fix}(\mathrm{S}) \neq \emptyset$;

- $\mathrm{V}: \mathrm{H}_{1} \rightarrow \mathrm{H}_{1}$ is l-Lipschitzian with constant $\mathrm{l} \in[0, \infty)$;

- $\mathrm{F}: \mathrm{H}_{1} \rightarrow \mathrm{H}_{1}$ is a $\mathrm{k}$-Lipschitzian and $\eta$-strongly monotone operator with constants $\mathrm{\kappa}>0$ and $\eta>0$;

- constants $\mu, \sigma, l, \tau$, and $\gamma$ satisfy $0<\mu<\frac{2 \eta}{\kappa^{2}}, 0<\sigma l<\tau=1-\sqrt{1-\mu\left(2 \eta-\mu \kappa^{2}\right)}$, and $0<\gamma<\frac{1}{\|A\|^{2}}$;

- $\Omega$ is the set of solutions of SCFPP (1.2). way:

First, we introduce the following iterative algorithm that generates a net $\left\{x_{\mathbf{t}}\right\}_{\mathbf{t} \in\left(0, \frac{1}{\tau-\sigma)}\right)}$ in an implicit

$$
x_{\mathrm{t}}=\mathrm{T}\left[\mathrm{I}-\gamma \mathrm{A}^{*}(\mathrm{I}-\mathrm{S}) \mathrm{A}\right] \mathrm{T}\left[\mathrm{t} \sigma \mathrm{V} \mathrm{x}_{\mathrm{t}}+(\mathrm{I}-\mathrm{t} \mu \mathrm{F}) \mathrm{x}_{\mathrm{t}}\right] .
$$

We prove strong convergence of $\left\{x_{t}\right\}$ as $t \rightarrow 0$ to a $x^{*}$ which is a solution of the following variational inequality:

$$
x^{*} \in \Omega \text { such that }\left\langle\sigma \mathrm{V}^{*}-\mu \mathrm{F} x^{*}, \widetilde{x}-\chi^{*}\right\rangle \leqslant 0, \quad \forall \widetilde{x} \in \Omega .
$$

Now, for $\mathrm{t} \in\left(0, \frac{1}{\tau-\sigma \mathrm{l}}\right)$, consider a mapping $\mathrm{W}_{\mathrm{t}}: \mathrm{C} \rightarrow \mathrm{C}$ defined by

$$
W_{t} x:=T\left[I-\gamma A^{*}(I-S) A\right] T[t \sigma V x+(I-t \mu F) x], \quad x \in C .
$$

It is easy to see that $W_{t}$ is a contractive mapping with constant $1-t(\tau-\sigma l)$. Indeed, note that $T$ and $\mathrm{I}-\gamma A^{*}(\mathrm{I}-\mathrm{S}) \mathrm{A}$ are nonexpansive (by Proposition 2.2). Thus, by Lemma 2.8, we have for $x, y \in \mathrm{C}$,

$$
\begin{aligned}
\left\|W_{\mathrm{t}} x-W_{\mathrm{t}} \mathrm{y}\right\| & =\left\|\mathrm{T}\left[\mathrm{I}-\gamma \mathrm{A}^{*}(\mathrm{I}-\mathrm{S}) \mathrm{A}\right] \mathrm{T}[\mathrm{t} \sigma \mathrm{V} \mathrm{x}+(\mathrm{I}-\mathrm{t} \mu \mathrm{F}) \mathrm{x}]-\mathrm{T}\left[\mathrm{I}-\gamma \mathrm{A}^{*}(\mathrm{I}-\mathrm{S}) \mathrm{A}\right] \mathrm{T}[\mathrm{t} \sigma \mathrm{V} y+(\mathrm{I}-\mathrm{t} \mu \mathrm{F}) \mathrm{y}]\right\| \\
& \leqslant \mathrm{t} \sigma\|\mathrm{V} x-\mathrm{V} \mathrm{y}\|+\|(\mathrm{I}-\mu \mathrm{tF}) \mathrm{x}-(\mathrm{I}-\mu \mathrm{tF}) \mathrm{y}\| \\
& \leqslant \mathrm{t} \sigma \mathrm{l}\|\mathrm{x}-\mathrm{y}\|+(1-\mathrm{t} \tau)\|x-\mathrm{y}\| \\
& =[1-\mathrm{t}(\tau-\sigma \mathrm{l})]\|x-\mathrm{y}\| .
\end{aligned}
$$

Therefore $W_{t}$ is a contractive mapping when $t \in\left(0, \frac{1}{\tau-\sigma l}\right)$. By the Banach contraction principle, $W_{t}$ has a unique fixed point in $C$, denoted by $x_{t}$, that is,

$$
x_{t}=T\left[I-\gamma A^{*}(I-S) A\right] T\left[t \sigma V x_{t}+(I-t \mu F) x_{t}\right],
$$

which is exactly (3.1).

We summarize the basic properties of $\left\{x_{t}\right\}$. 
Proposition 3.1. Let $\Omega \neq \emptyset$, and let $\left\{x_{\mathrm{t}}\right\}$ be defined via (3.1). Then

(i) $\left\{x_{\mathrm{t}}\right\}$ is bounded for $\mathrm{t} \in\left(0, \frac{1}{\tau-\sigma \mathrm{l}}\right)$;

(ii) $\lim _{\mathrm{t} \rightarrow 0}\left\|\mathrm{x}_{\mathrm{t}}-\mathrm{T}\left[\mathrm{I}-\gamma \mathrm{A}^{*}(\mathrm{I}-\mathrm{S}) \mathrm{A}\right] \mathrm{T} \mathrm{x}_{\mathrm{t}}\right\|=0$;

(iii) $x_{t}$ defines a continuous path from $\left(0, \frac{1}{\tau-\sigma l}\right)$ into $\mathrm{H}_{1}$.

Proof.

(i) Let $\tilde{x}$ be any point in $\Omega$. Then $\tilde{x} \in \operatorname{Fix}(T)$ and $A \tilde{x} \in \operatorname{Fix}(S)$. Set

$$
\mathrm{U}=\mathrm{I}-\gamma A^{*}(\mathrm{I}-\mathrm{S}) \mathrm{A} .
$$

Then, from Proposition 2.2 (iii), we have $\widetilde{x} \in \operatorname{Fix}(U)$, and we can rewrite (3.1) as

$$
x_{t}=\operatorname{TUT}\left[\mathrm{t} \sigma \mathrm{V} x_{\mathrm{t}}+(\mathrm{I}-\mathrm{t} \mu \mathrm{F}) \mathrm{x}_{\mathrm{t}}\right], \quad \mathrm{t} \in\left(0, \frac{1}{\tau-\sigma \mathrm{l}}\right) .
$$

It follows that

$$
\begin{aligned}
\left\|x_{t}-\widetilde{x}\right\| & =\left\|\operatorname{TUT}\left[\mathrm{t} \sigma V x_{t}+(I-t \mu F) x_{t}\right]-\widetilde{x}\right\| \\
& \leqslant\left\|t \sigma\left(V x_{t}-V \widetilde{x}\right)\right\|+\left\|(I-t \mu F) x_{t}-(I-t \mu F) \widetilde{x}\right\|+\|t \sigma V \widetilde{x}-t \mu F \widetilde{x}\| \\
& \leqslant t \sigma\left\|x_{t}-\widetilde{x}\right\|+(1-t \tau)\left\|x_{t}-\widetilde{x}\right\|+t\|\sigma \sqrt{x}-\mu F \tilde{x}\| \\
& =[1-(\tau-\sigma l) t]\left\|x_{t}-\widetilde{x}\right\|+t\|\sigma \sqrt{x}-\mu F \tilde{x}\| .
\end{aligned}
$$

Hence

$$
\left\|x_{t}-\tilde{x}\right\| \leqslant \frac{1}{\tau-\sigma l}\|\sigma \sqrt{x}-\mu F \tilde{x}\| .
$$

Then $\left\{x_{t}\right\}$ is bounded and so are $\left\{\mathrm{V} x_{t}\right\},\left\{\mathrm{U} x_{t}\right\}$, and $\left\{F x_{t}\right\}$.

(ii) From (3.1), we have

$$
\left\|x_{t}-T\left[I-\gamma A^{*}(I-S) A\right] T x_{t}\right\|=\left\|\operatorname{TUT}\left[t \sigma V x_{t}+(I-t \mu F) x_{t}\right]-T U T x_{t}\right\| \leqslant t\left\|\sigma V x_{t}-\mu F x_{t}\right\| .
$$

By boundedness of $\left\{\mathrm{V} x_{t}\right\}$ and $\left\{F x_{t}\right\}$, we obtain

$$
\lim _{t \rightarrow 0}\left\|x_{t}-T\left[I-\gamma A^{*}(I-S) A\right] T x_{t}\right\|=0 .
$$

(iii) Let $t, t_{0} \in\left(0, \frac{1}{\tau-\sigma l}\right)$. We calculate

$$
\begin{aligned}
\left\|x_{\mathrm{t}}-x_{\mathrm{t}_{0}}\right\|= & \left\|\mathrm{T}\left[\mathrm{I}-\gamma \mathrm{A}^{*}(\mathrm{I}-\mathrm{S}) \mathrm{A}\right] \mathrm{T}\left[\mathrm{t} \sigma \mathrm{V} \mathrm{x}_{\mathrm{t}}+(\mathrm{I}-\mathrm{t} \mu \mathrm{F}) \mathrm{x}_{\mathrm{t}}\right]-\mathrm{T}\left[\mathrm{I}-\gamma \mathrm{A}^{*}(\mathrm{I}-\mathrm{S}) \mathrm{A}\right] \mathrm{T}\left[\mathrm{t}_{0} \sigma \mathrm{V} \mathrm{x}_{\mathrm{t}_{0}}+(\mathrm{I}-\mathrm{t} \mu \mathrm{F}) \mathrm{x}_{\mathrm{t}_{0}}\right]\right\| \\
\leqslant & \left\|\mathrm{t} \sigma \mathrm{V} \mathrm{x}_{\mathrm{t}}+(\mathrm{I}-\mathrm{t} \mu \mathrm{F}) \mathrm{x}_{\mathrm{t}}-\left(\mathrm{t}_{0} \sigma \mathrm{V} \mathrm{x}_{\mathrm{t}_{0}}+(\mathrm{I}-\mathrm{t} \mu \mathrm{F}) \mathrm{x}_{\mathrm{t}_{0}}\right)\right\| \\
\leqslant & \left\|\mathrm{t} \sigma \mathrm{V} \mathrm{x}_{\mathrm{t}}-\mathrm{t}_{0} \sigma \mathrm{V} \mathrm{x}_{\mathrm{t}}\right\|+\left\|(\mathrm{I}-\mathrm{t} \mu \mathrm{F}) \mathrm{x}_{\mathrm{t}}-(\mathrm{I}-\mathrm{t} \mu \mathrm{F}) \mathrm{x}_{\mathrm{t}_{0}}\right\| \\
& \quad+\left\|\mathrm{t}_{0} \sigma \mathrm{V} \mathrm{x}_{\mathrm{t}}-\mathrm{t}_{0} \sigma \mathrm{V} \mathrm{x}_{\mathrm{t}_{0}}\right\|+\left\|(\mathrm{I}-\mathrm{t} \mu \mathrm{F}) \mathrm{x}_{\mathrm{t}_{0}}-\left(\mathrm{I}-\mathrm{t}_{0} \mu \mathrm{F}\right) \mathrm{x}_{\mathrm{t}_{0}}\right\| \\
\leqslant & \sigma\left\|\mathrm{V} \mathrm{x}_{\mathrm{t}}\right\|\left|\mathrm{t}-\mathrm{t}_{0}\right|+(1-\mathrm{t} \tau)\left\|\mathrm{x}_{\mathrm{t}}-\mathrm{x}_{\mathrm{t}_{0}}\right\|+\mathrm{t}_{0} \sigma \mathrm{l}\left\|\mathrm{x}_{\mathrm{t}}-\mathrm{x}_{\mathrm{t}_{0}}\right\|+\mu\left\|\mathrm{F} \mathrm{x}_{\mathrm{t}_{0}}\right\|\left|\mathrm{t}-\mathrm{t}_{0}\right| .
\end{aligned}
$$

This implies that

$$
\left\|x_{t}-x_{t_{0}}\right\| \leqslant \frac{\sigma\left\|V x_{t}\right\|+\mu\left\|F x_{t_{0}}\right\|}{t \tau-t_{0} \sigma l}\left|t-t_{0}\right|
$$

This completes the proof.

Theorem 3.2. Let $\Omega \neq \emptyset$, and let the net $\left\{x_{\mathrm{t}}\right\}$ be defined via (3.1). Then $x_{\mathrm{t}}$ converges strongly to a point $x^{*}$ as $t \rightarrow 0$, which solves the variational inequality (3.2). 
Proof. First, we show easily the uniqueness of a solution of the variational inequality (3.2). In fact, noting that $0 \leqslant \sigma l<\tau$ and $\mu \eta \geqslant \tau \Longleftrightarrow k \geqslant \eta$, it follows that

$$
\langle(\mu \mathrm{F}-\sigma \mathrm{V}) x-(\mu \mathrm{F}-\sigma \mathrm{V}) \mathrm{y}, x-\mathrm{y}\rangle \geqslant(\mu \eta-\sigma l)\|x-y\|^{2} .
$$

That is, $\mu \mathrm{F}-\sigma \mathrm{V}$ is strongly monotone for $0 \leqslant \sigma l<\tau \leqslant \mu \eta$. So the variational inequality (3.2) has only one solution.

Next, we show that $\left\{x_{t}\right\}$ is relatively norm-compact as $t \rightarrow 0^{+}$. To this end, set $U=I-\gamma A^{*}(I-S) A$ and let $\widetilde{x}$ be any point in $\Omega$. Then $\widetilde{x} \in \operatorname{Fix}(T), A \widetilde{x} \in \operatorname{Fix}(S)$, and $\widetilde{x} \in \operatorname{Fix}(U)$ (by Proposition 2.2 (iii)). Let $\left\{t_{n}\right\} \subset\left(0, \frac{1}{\tau-\sigma l}\right)$ be such that $t_{n} \rightarrow 0$ as $n \rightarrow \infty$. Put $x_{n}:=x_{t_{n}}$. From Proposition 3.1 (ii), we have

$$
\lim _{n \rightarrow \infty}\left\|x_{n}-\operatorname{TUT}_{n}\right\|=0 \text {. }
$$

Put $z_{\mathrm{t}}=\mathrm{t} \sigma \mathrm{V} \mathrm{x}_{\mathrm{t}}+(\mathrm{I}-\mathrm{t} \mu \mathrm{F}) \mathrm{x}_{\mathrm{t}}, \mathrm{y}_{\mathrm{t}}=\mathrm{T}\left[\mathrm{t} \sigma \mathrm{V} \mathrm{x}_{\mathrm{t}}+(\mathrm{I}-\mathrm{t} \mu \mathrm{F}) \mathrm{x}_{\mathrm{t}}\right]=\mathrm{T} z_{\mathrm{t}}, z_{\mathrm{n}}:=z_{\mathrm{t}_{\mathrm{n}}}$, and $\mathrm{y}_{\mathrm{n}}:=\mathrm{y}_{\mathrm{t}_{\mathrm{n}}}=\mathrm{T} z_{\mathrm{n}}$. Then we have, for any $\tilde{x} \in \Omega$,

$$
y_{t}-\widetilde{x}=y_{t}-z_{t}+z_{t}-\widetilde{x}=y_{t}-z_{t}+t \sigma\left(V x_{t}-\sqrt{x}\right)+(I-t \mu F) x_{t}-(I-t \mu F) \widetilde{x}+t(\sigma \sqrt{x}-\mu F \widetilde{x}) .
$$

Since $T$ is a firmly nonexpansive mapping with a fixed point $\widetilde{x}$, we have

$$
\left\langle y_{t}-z_{t}, y_{t}-\tilde{x}\right\rangle \leqslant 0 .
$$

Combining (3.4) with (3.5) along with Lemma 2.8, we get

$$
\begin{aligned}
\left\|y_{t}-\widetilde{x}\right\|^{2}= & \left\langle y_{t}-\widetilde{x}, y_{t}-\widetilde{x}\right\rangle \\
= & \left\langle y_{t}-z_{t}, y_{t}-\widetilde{x}\right\rangle+t \sigma\left\langle V x_{t}-\sqrt{x}, y_{t}-\widetilde{x}\right\rangle \\
& +\left\langle(I-t \mu F) x_{t}-(I-t \mu F) \widetilde{x}, y_{t}-\widetilde{x}\right\rangle+t\left\langle\sigma \sqrt{x}-\mu F \widetilde{x}, y_{t}-\widetilde{x}\right\rangle \\
\leqslant & t \sigma l\left\|x_{t}-\widetilde{x}\right\|\left\|y_{t}-\widetilde{x}\right\|+(1-t \tau)\left\|x_{t}-\widetilde{x}\right\|\left\|y_{t}-\widetilde{x}\right\|+t\left\langle\sigma \sqrt{x}-\mu F \widetilde{x}, y_{t}-\widetilde{x}\right\rangle \\
= & {[1-(\tau-\sigma l) t]\left\|x_{t}-\widetilde{x}\right\|\left\|y_{t}-\widetilde{x}\right\|+t\left\langle\sigma \sqrt{x}-\mu F \widetilde{x}, y_{t}-\widetilde{x}\right\rangle } \\
\leqslant & \frac{1-(\tau-\sigma l) t}{2}\left\|x_{t}-\widetilde{x}\right\|^{2}+\frac{1}{2}\left\|y_{t}-\widetilde{x}\right\|^{2}+t\left\langle\sigma \sqrt{x}-\mu F \widetilde{x}, y_{t}-\widetilde{x}\right\rangle .
\end{aligned}
$$

It follows that

$$
\left\|y_{t}-\tilde{x}\right\|^{2} \leqslant[1-(\tau-\sigma l) t]\left\|x_{t}-\tilde{x}\right\|^{2}+2 t\left\langle\sigma V \widetilde{x}-\mu F \widetilde{x}, y_{t}-\widetilde{x}\right\rangle .
$$

Thus, from Proposition 2.2, we have

$$
\begin{aligned}
\left\|x_{t}-\tilde{x}\right\|^{2}=\left\|T U T z_{t}-T U T \tilde{x}\right\|^{2} & \leqslant\left\|T U y_{t}-\operatorname{TU} \widetilde{x}\right\|^{2} \\
& \leqslant\left\|y_{t}-\tilde{x}\right\|^{2} \leqslant[1-(\tau-\sigma l) t]\left\|x_{t}-\widetilde{x}\right\|^{2}+2 t\left\langle\sigma \sqrt{x}-\mu F \widetilde{x}, y_{t}-\widetilde{x}\right\rangle .
\end{aligned}
$$

Hence, we obtain

$$
\left\|x_{t}-\tilde{x}\right\|^{2} \leqslant \frac{2}{\tau-\sigma l}\left\langle\sigma \sqrt{x}-\mu F \tilde{x}, y_{t}-\widetilde{x}\right\rangle
$$

In particular, we have

$$
\left\|x_{n}-\tilde{x}\right\|^{2} \leqslant \frac{2}{\tau-\sigma l}\left\langle\sigma \sqrt{x}-\mu F \tilde{x}, y_{n}-\tilde{x}\right\rangle, \quad \tilde{x} \in \Omega
$$

Note that

$$
\left\|x_{t}-z_{t}\right\|=\left\|x_{t}-\left[t \sigma V x_{t}+(I-t \mu F) x_{t}\right]\right\| \leqslant t\left\|\sigma V x_{t}-\mu F x_{t}\right\| \rightarrow 0 \quad \text { as } t \rightarrow 0
$$

So,

$$
\lim _{n \rightarrow \infty}\left\|x_{n}-z_{n}\right\|=0
$$


Observe that

$$
\begin{aligned}
\left\|z_{n}-\tilde{x}\right\| & =\left\|t_{n} \sigma V x_{n}+\left(I-t_{n} \mu F\right) x_{n}-\tilde{x}\right\| \\
& =\left\|\left(x_{n}-\tilde{x}\right)+t_{n}\left(\sigma V x_{n}-\mu F x_{n}\right)\right\| \\
& \leqslant\left\|x_{n}-\tilde{x}\right\|+t_{n}\left\|\sigma V x_{n}-\mu F x_{n}\right\| .
\end{aligned}
$$

Then, since every firmly nonexpansive mapping with a fixed point is firmly quasi-nonexpansive, from (3.8) we deduce

$$
\begin{aligned}
\left\|x_{n}-\tilde{x}\right\|^{2} & =\| T U T z_{n}-\text { TUT } \tilde{x} \|^{2} \\
& \leqslant\left\|T z_{n}-\widetilde{x}\right\|^{2} \\
& \leqslant\left\|z_{n}-\widetilde{x}\right\|^{2}-\left\|T z_{n}-z_{n}\right\|^{2} \\
& \leqslant\left(\left\|x_{n}-\widetilde{x}\right\|+t_{n}\left\|\sigma V x_{n}-\mu F x_{n}\right\|\right)^{2}-\left\|T z_{n}-z_{n}\right\|^{2} \\
& \leqslant\left\|x_{n}-\widetilde{x}\right\|^{2}+t_{n} M-\left\|T z_{n}-z_{n}\right\|^{2},
\end{aligned}
$$

where $M>0$ is an appropriate constant. This implies that

$$
\lim _{n \rightarrow \infty}\left\|y_{n}-z_{n}\right\|=\lim _{n \rightarrow \infty}\left\|T z_{n}-z_{n}\right\|=0 .
$$

Since $\left\{x_{n}\right\}$ is bounded, there exists a subsequence $\left\{x_{n_{i}}\right\}$ of $\left\{x_{n}\right\}$ which converges weakly to a point $x^{*}$. Without loss of generality, we may assume that $\left\{x_{n}\right\}$ converges weakly to $x^{*}$. Then by (3.7) and (3.9), $y_{n} \rightarrow x^{*}$. Noticing (3.3), we can use Lemma 2.4 to get $x^{*}=$ TUT $x^{*}$. By Proposition 2.1 (iv), we have $\mathrm{T} x^{*}=x^{*}$ and $U x^{*}=x^{*}$, and hence $S\left(A x^{*}\right)=A x^{*}$. Thus $x^{*} \in \operatorname{Fix}(T)$ and $A x^{*} \in \operatorname{Fix}(S)$, that is, $x^{*} \in \Omega$. Therefore, we can substitute $x^{*}$ for $\widetilde{x}$ in (3.6) to obtain

$$
\left\|x_{n}-x^{*}\right\|^{2} \leqslant \frac{2}{\tau-\sigma l}\left\langle\sigma V x^{*}-\mu F x^{*}, y_{n}-x^{*}\right\rangle .
$$

Consequently, $y_{n} \rightarrow x^{*}$ actually implies that $x_{n} \rightarrow x^{*}$. This proves the relative norm-compactness of the net $\left\{x_{t}\right\}$ as $t \rightarrow 0^{+}$.

Letting $n \rightarrow \infty$ in (3.6), we have

$$
\left\|x^{*}-\tilde{x}\right\|^{2} \leqslant \frac{2}{\tau-\sigma l}\left\langle\sigma \sqrt{x}-\mu F \widetilde{x}, x^{*}-\tilde{x}\right\rangle, \quad \tilde{x} \in \Omega .
$$

This implies that $\chi^{*} \in \Omega$ solves the variational inequality

$$
\left\langle\sigma \sqrt{x}-\mu F \tilde{x}, \widetilde{x}-x^{*}\right\rangle \leqslant 0, \quad \tilde{x} \in \Omega .
$$

By Lemma 2.5, (3.10) is equivalent to its dual variational inequality

$$
\left\langle\sigma \mathrm{V} x^{*}-\mu \mathrm{F} x^{*}, \tilde{x}-x^{*}\right\rangle \leqslant 0, \quad \tilde{x} \in \Omega .
$$

This is exactly (3.2). By uniqueness of the solution of the variational inequality (3.2), we deduce that each cluster point of $\left\{x_{t}\right\}$ as $t \rightarrow 0^{+}$equals to $x^{*}$. Therefore $x_{t} \rightarrow x^{*}$ as $t \rightarrow 0^{+}$. This completes the proof.

Taking $\mathrm{F}=\mathrm{I}$ and $\mu=1$ in Theorem 3.2, we have the following corollary.

Corollary 3.3. Let the net $\left\{x_{t}\right\}$ be defined by

$$
x_{t}=T\left[I-\gamma A^{*}(I-S) A\right] T\left[t \sigma V x_{t}+(1-t) x_{t}\right], \quad t \in\left(0, \frac{1}{1-\sigma l}\right) .
$$

Then $\left\{x_{\mathrm{t}}\right\}$ converges strongly as $\mathrm{t} \rightarrow 0$ to a point $x^{*}$ which is the unique solution of variational inequality

$$
x^{*} \in \Omega \quad \text { such that }\left\langle\sigma \vee x^{*}-x^{*}, \widetilde{x}-x^{*}\right\rangle \leqslant 0, \quad \forall \widetilde{x} \in \Omega .
$$

Taking $\mathrm{V}=0$ in (3.11), we get the following corollary. 
Corollary 3.4. Let the net $\left\{x_{\mathrm{t}}\right\}$ be defined by

$$
x_{\mathrm{t}}=\mathrm{T}\left[\mathrm{I}-\gamma \mathrm{A}^{*}(\mathrm{I}-\mathrm{S}) \mathrm{A}\right] \mathrm{T}\left[(1-\mathrm{t}) \mathrm{x}_{\mathrm{t}}\right], \quad \mathrm{t} \in(0,1) .
$$

Then $\left\{x_{\mathrm{t}}\right\}$ converges strongly as $\mathrm{t} \rightarrow 0$ to a point $x^{*}$ which is the minimum norm solution of the SCFPP (1.2).

Proof. If we take $V=0$, then (3.11) reduces to (3.12). Thus, $x_{t} \rightarrow x^{*} \in \Omega$ which satisfies

$$
\left\langle-x^{*}, \widetilde{x}-x^{*}\right\rangle \leqslant 0, \quad \forall \widetilde{x} \in \Omega \text {. }
$$

Thus

$$
\left\|x^{*}\right\|^{2} \leqslant\left\langle x^{*}, \widetilde{x}\right\rangle \leqslant\left\|x^{*}\right\|\|\widetilde{x}\|, \quad \forall \widetilde{x} \in \Omega,
$$

which implies $\left\|x^{*}\right\| \leqslant\|\widetilde{x}\|$ for all $\widetilde{x} \in \Omega$. That is, $x^{*}$ is the minimum norm solution of the SCFPP (1.2). This completes the proof.

Next, we propose the following iterative algorithm which generates a sequence in an explicit way:

$$
x_{n+1}=T\left[I-\gamma A^{*}(I-S) A\right] T\left[\alpha_{n} \sigma V x_{n}+\left(I-\alpha_{n} \mu F\right) x_{n}\right], \quad n \geqslant 0,
$$

where $\left\{\alpha_{n}\right\} \subset[0,1]$ and $x_{0} \in H_{1}$ is an arbitrary initial guess, and establishes strong convergence of this sequence to a point $x^{*}$, which is also a solution of the variational inequality (3.2).

Theorem 3.5. Let $\Omega \neq \emptyset$, and let $\left\{x_{n}\right\}$ be the sequence generated by the explicit algorithm (3.13), where $\left\{\alpha_{n}\right\}$ satisfies the following conditions:

(C1) $\left\{\alpha_{n}\right\} \subset[0,1], \lim _{n \rightarrow \infty} \alpha_{n}=0$;

(C2) $\sum_{n=0}^{\infty} \alpha_{n}=\infty$.

Then $\left\{x_{n}\right\}$ converges strongly to a point $x^{*} \in \Omega$ as $\mathrm{n} \rightarrow \infty$, which solves the variational inequality (3.2).

Proof. Let $\widetilde{x} \in \Omega$ and let $\mathrm{U}=\mathrm{I}-\gamma A^{*}(\mathrm{I}-\mathrm{S}) A$. Then (3.13) becomes

$$
x_{n+1}=\operatorname{TUT}\left[\alpha_{n} \sigma V x_{n}+\left(I-\alpha_{n} \mu F\right) x_{n}\right], \quad n \geqslant 0 .
$$

We divide the proof into the following steps:

Step 1. We show that $\left\{x_{n}\right\}$ is bounded. In fact, from (3.13), we deduce

$$
\begin{aligned}
\left\|x_{n+1}-\tilde{x}\right\| & =\left\|\operatorname{TUT}\left[\alpha_{n} \sigma V x_{n}+\left(I-\alpha_{n} \mu F\right) x_{n}\right]-T U T \widetilde{x}\right\| \\
& \leqslant\left\|\alpha_{n} \sigma V x_{n}+\left(I-\alpha_{n} \mu F\right) x_{n}-\tilde{x}\right\| \\
& \leqslant \alpha_{n} \sigma\left\|V x_{n}-\sqrt{x}\right\|+\left\|\left(I-\alpha_{n} \mu F\right) x_{n}-\left(I-\alpha_{n} \mu F\right) \widetilde{x}\right\|+\alpha_{n}\|\sigma \sqrt{x}-\mu F \widetilde{x}\| \\
& \leqslant \alpha_{n} l\left\|x_{n}-\widetilde{x}\right\|+\left(1-\alpha_{n} \tau\right)\left\|x_{n}-\widetilde{x}\right\|+\alpha_{n}\|\sigma \sqrt{x}-\mu F \widetilde{x}\| \\
& =\left[1-(\tau-\sigma l) \alpha_{n}\right]\left\|x_{n}-\tilde{x}\right\|+(\tau-\sigma l) \alpha_{n} \frac{\|\sigma \sqrt{x}-\mu F \widetilde{x}\|}{\tau-\sigma l} .
\end{aligned}
$$

It follows by induction that

$$
\left\|x_{n+1}-\widetilde{x}\right\| \leqslant \max \left\{\left\|x_{n}-\widetilde{x}\right\|, \frac{\|\sigma \sqrt{x}-\mu F \tilde{x}\|}{\tau-\sigma l}\right\} \leqslant \cdots \leqslant \max \left\{\left\|x_{0}-\widetilde{x}\right\|, \frac{\|\sigma \sqrt{x}-\mu F \tilde{x}\|}{\tau-\sigma l}\right\} .
$$

This means that $\left\{x_{n}\right\}$ is bounded. It is easy to deduce that $\left\{V x_{n}\right\},\left\{U x_{n}\right\}$ and $\left.F x_{n}\right\}$ are also bounded.

Step 2. We show that $\lim _{\mathfrak{n} \rightarrow \infty}\left\|\mathrm{TUT} z_{\mathfrak{n}}-z_{\mathfrak{n}}\right\|=0$. To this end, set

$$
y_{n}:=T\left[\alpha_{n} \sigma V x_{n}+\left(I-\alpha_{n} \mu F\right) x_{n}\right]
$$

and

$$
z_{n}:=\alpha_{n} \sigma V x_{n}+\left(I-\alpha_{n} \mu F\right) x_{n}, \quad n \geqslant 0 .
$$

Since $U$ is averaged by Proposition 2.2 (ii) and also, every firmly nonexpansive mapping is averaged, thus 
T is averaged. Since the composite of finitely many averaged mappings is averaged by Proposition 2.1 (c), TU is averaged. Hence, there exists a positive constant $\lambda_{1} \in(0,1)$ such that $T U=\left(1-\lambda_{1}\right) I+\lambda_{1} G_{1}$, where $G_{1}$ is a nonexpansive mapping. Since $T$ is averaged, there exists $\lambda_{2} \in(0,1)$ such that $T=\left(1-\lambda_{2}\right) I+\lambda_{2} G_{2}$, where $G_{2}$ is a nonexpansive mapping. It follows that

$$
\begin{aligned}
y_{n} & =T z_{n} \\
& =\left(\left(1-\lambda_{2}\right) I+\lambda_{2} G_{2}\right) z_{n} \\
& =\left(\left(1-\lambda_{2}\right) I+\lambda_{2} G_{2}\right)\left(\alpha_{n} \sigma V x_{n}+\left(I-\alpha_{n} \mu F\right) x_{n}\right) \\
& =\left(1-\lambda_{2}\right)\left(\alpha_{n} \sigma V x_{n}+\left(I-\alpha_{n} \mu F\right) x_{n}\right)+\lambda_{z} G_{2} z_{n} \\
& =\left(1-\lambda_{2}\right)\left(x_{n}+\alpha_{n}\left(\sigma V x_{n}-\mu F x_{n}\right)\right)+\lambda_{2} G_{2} z_{n} \\
& =\left(1-\lambda_{2}\right) x_{n}+\lambda_{2}\left[\frac{\left(1-\lambda_{2}\right)}{\lambda_{2}} \alpha_{n}\left(\sigma V x_{n}-\mu F x_{n}\right)+G_{2} z_{n}\right]=\left(1-\lambda_{2}\right) x_{n}+\lambda_{2} q_{n},
\end{aligned}
$$

where

$$
q_{n}=\frac{\left(1-\lambda_{2}\right)}{\lambda_{2}} \alpha_{n}\left(\sigma V x_{n}-\mu F x_{n}\right)+G_{2} z_{n}
$$

Moreover, we get

$$
\begin{aligned}
& \left\|q_{n+1}-q_{n}\right\| \\
& \left.\quad=\| \frac{\left(1-\lambda_{2}\right)}{\lambda_{2}} \alpha_{n+1}\left(\sigma V x_{n+1}-\mu F x_{n+1}\right)+G_{2} z_{n+1}\right)-\frac{\left(1-\lambda_{2}\right)}{\lambda_{2}} \alpha_{n}\left(\sigma V x_{n}-\mu F x_{n}\right)-G_{2} z_{n} \| \\
& \quad \leqslant\left\|G_{2} z_{n+1}-G_{2} z_{n}\right\|+\frac{\left(1-\lambda_{2}\right)}{\lambda_{2}}\left[\alpha_{n+1}\left\|\sigma V x_{n+1}-\mu F x_{n+1}\right\|+\alpha_{n}\left\|\sigma V x_{n}-\mu F x_{n}\right\|\right] \\
& \quad \leqslant\left\|z_{n+1}-z_{n}\right\|+\frac{\left(1-\lambda_{2}\right)}{\lambda_{2}}\left[\alpha_{n+1}\left\|\sigma V x_{n+1}-\mu F x_{n+1}\right\|+\alpha_{n}\left\|\left(\sigma V x_{n}-\mu F x_{n}\right)\right\|\right] .
\end{aligned}
$$

In view of (3.13) and (3.14), we have

$$
\begin{aligned}
x_{n+1} & =T_{y_{n}} \\
& =\left(\left(1-\lambda_{1}\right) I+\lambda-1 G_{1}\right) y_{n} \\
& =\left(1-\lambda_{1}\right) y_{n}+\lambda_{1} G_{1} y_{n} \\
& =\left(1-\lambda_{1}\right)\left[\left(1-\lambda_{2}\right) x_{n}+\lambda_{2} q_{n}\right]+\lambda_{1} G_{1} y_{n} \\
& =\left(1-\lambda_{1}\right)\left(1-\lambda_{2}\right) x_{n}+\left(1-\lambda_{1}\right) \lambda_{2} q_{n}+\lambda_{1} G_{1} y_{n} \\
& =\left(1-\left(\lambda_{1}+\lambda_{2}-\lambda_{1} \lambda_{2}\right)\right) x_{n}+\left(1-\lambda_{1}\right) \lambda_{2} q_{n}+\lambda_{1} G_{1} y_{n} \\
& =\left(1-\lambda_{3}\right) x_{n}+\lambda_{3}\left[\frac{\left(1-\lambda_{1}\right) \lambda_{2}}{\lambda_{3}} q_{n}+\frac{\lambda_{1}}{\lambda_{3}} G_{1} y_{n}\right] \\
& =\left(1-\lambda_{3}\right) x_{n}+\lambda_{3} p_{n},
\end{aligned}
$$

where $\lambda_{3}=\lambda_{1}+\lambda_{2}-\lambda_{1} \lambda_{2}$ and $p_{n}=\frac{\left(1-\lambda_{1}\right) \lambda_{2}}{\lambda_{3}} q_{n}+\frac{\lambda_{1}}{\lambda_{3}} G_{1} y_{n}$. Thus, from (3.15), we derive

$$
\begin{aligned}
\left\|p_{n+1}-p_{n}\right\| & =\left\|\frac{\left(1-\lambda_{1}\right) \lambda_{2}}{\lambda_{3}} q_{n+1}+\frac{\lambda_{1}}{\lambda_{3}} G_{1} y_{n+1}-\frac{\left(1-\lambda_{1}\right) \lambda_{2}}{\lambda_{3}} q_{n}-\frac{\lambda_{1}}{\lambda_{3}} G_{1} y_{n}\right\| \\
& =\left\|\frac{\left(1-\lambda_{1}\right) \lambda_{2}}{\lambda_{3}}\left(q_{n+1}-q_{n}\right)+\frac{\lambda_{1}}{\lambda_{3}}\left(G_{1} y_{n+1}-G_{1} y_{n}\right)\right\| \\
& \leqslant \frac{\left(1-\lambda_{1}\right) \lambda_{2}}{\lambda_{3}}\left\|q_{n+1}-q_{n}\right\|+\frac{\lambda_{1}}{\lambda_{3}}\left\|G_{1} y_{n+1}-G_{1} y_{n}\right\| \\
& \leqslant \frac{\left(1-\lambda_{1}\right) \lambda_{2}}{\lambda_{3}}\left\|q_{n+1}-q_{n}\right\|+\frac{\lambda_{1}}{\lambda_{3}}\left\|y_{n+1}-y_{n}\right\|
\end{aligned}
$$




$$
\begin{aligned}
= & \frac{\left(1-\lambda_{1}\right) \lambda_{2}}{\lambda_{3}}\left\|q_{n+1}-q_{n}\right\|+\frac{\lambda_{1}}{\lambda_{3}}\left\|T z_{n+1}-T z_{n}\right\| \\
\leqslant & \frac{\left(1-\lambda_{1}\right) \lambda_{2}}{\lambda_{3}}\left\|q_{n+1}-q_{n}\right\|+\frac{\lambda_{1}}{\lambda_{3}}\left\|z_{n+1}-z_{n}\right\| \\
\leqslant & \frac{\left(1-\lambda_{1}\right) \lambda_{2}}{\lambda_{3}}\left\|z_{n+1}-z_{n}\right\| \\
& +\frac{\left(1-\lambda_{1}\right)\left(1-\lambda_{2}\right)}{\lambda_{3}}\left[\alpha_{n+1}\left\|\sigma V x_{n+1}-\mu F x_{n+1}\right\|+\alpha_{n}\left\|\sigma V x_{n}-\mu F x_{n}\right\|\right]+\frac{\lambda_{1}}{\lambda_{3}}\left\|z_{n+1}-z_{n}\right\| \\
= & \frac{\lambda_{1}+\lambda_{2}-\lambda_{1} \lambda_{2}}{\lambda_{3}}\left\|z_{n+1}-z_{n}\right\| \\
& +\frac{\left(1-\lambda_{3}\right)}{\lambda_{3}}\left[\alpha_{n+1}\left\|\sigma V x_{n+1}-\mu F x_{n+1}\right\|+\alpha_{n}\left\|\sigma V x_{n}-\mu F x_{n}\right\|\right] \\
= & \left\|z_{n+1}-z_{n}\right\|+\frac{\left(1-\lambda_{3}\right)}{\lambda_{3}}\left[\alpha_{n+1}\left\|\sigma V x_{n+1}-\mu F x_{n+1}\right\|+\alpha_{n}\left\|\sigma V x_{n}-\mu F x_{n}\right\|\right] \\
= & \|\left(\alpha_{n+1} \sigma V x_{n+1}+\left(I-\alpha_{n+1} \mu F\right) x_{n+1}-\left(\alpha_{n} \sigma V x_{n}+\left(I-\alpha_{n} \mu F x_{n}\right) n \|\right.\right. \\
& +\frac{\left(1-\lambda_{3}\right)}{\lambda_{3}}\left[\alpha_{n+1}\left\|\left(\sigma V x_{n+1}-\mu F x_{n+1}\right)\right\|+\alpha_{n}\left\|\left(\sigma V x_{n}-\mu F x_{n}\right)\right\|\right] \\
= & \left\|\left(x_{n+1}-x_{n}\right)+\alpha_{n+1}\left(\sigma V x_{n+1}-\mu F x_{n+1}\right)-\alpha_{n}\left(\sigma V x_{n}-\mu F x_{n}\right)\right\| \\
& +\frac{\left(1-\lambda_{3}\right)}{\lambda_{3}}\left[\alpha_{n+1}\left\|\sigma V x_{n+1}-\mu F x_{n+1}\right\|+\alpha_{n}\left\|\sigma V x_{n}-\mu F x_{n}\right\|\right] \\
\leqslant & \left\|x_{n+1}-x_{n}\right\|+\alpha_{n+1}\left\|\sigma V x_{n+1}-\mu F x_{n+1}\right\|+\alpha_{n}\left\|\sigma V x_{n}-\mu F x_{n}\right\| \\
& +\frac{\left(1-\lambda_{3}\right)}{\lambda_{3}}\left[\alpha_{n+1}\left\|\sigma V x_{n+1}-\mu F x_{n+1}\right\|+\alpha_{n}\left\|\sigma V x_{n}-\mu F x_{n}\right\|\right] . \\
&
\end{aligned}
$$

This implies that

$$
\begin{aligned}
\left\|p_{n+1}-p_{n}\right\|-\left\|x_{n+1}-x_{n}\right\| \leqslant & \alpha_{n+1}\left\|\sigma V x_{n+1}-\mu F x_{n+1}\right\|+\alpha_{n}\left\|\sigma V x_{n}-\mu F x_{n}\right\| \\
& +\frac{\left(1-\lambda_{3}\right)}{\lambda_{3}}\left[\alpha_{n+1}\left\|\sigma V x_{n+1}\right\|-\mu F x_{n+1}\left\|+\alpha_{n}\right\| \sigma V x_{n}-\mu F x_{n} \|\right]
\end{aligned}
$$

and

$$
\limsup _{n \rightarrow \infty}\left(\left\|p_{n+1}-p_{n}\right\|-\left\|x_{n+1}-x_{n}\right\|\right) \leqslant 0 .
$$

Thus, from (3.16), (3.17), and Lemma 2.6, we obtain

$$
\lim _{n \rightarrow \infty}\left\|p_{n}-x_{n}\right\|=0 .
$$

Also, by (3.16) and (3.18), we get

$$
\lim _{n \rightarrow \infty}\left\|x_{n+1}-x_{n}\right\|=\lambda_{3} \lim _{n \rightarrow \infty}\left\|p_{n}-x_{n}\right\|=0,
$$

and

$$
\lim _{n \rightarrow \infty}\left\|z_{n}-x_{n}\right\|=\lim _{n \rightarrow \infty} \alpha_{n}\left\|\sigma V x_{n}-\mu F x_{n}\right\|=0 .
$$

Therefore, from (3.19) and (3.20), we have

$$
\begin{aligned}
& \left\|T U T z_{n}-z_{n}\right\|=\left\|T U T z_{n}-x_{n}+x_{n}-z_{n}\right\| \\
& \leqslant \| \text { TUT } z_{n}-x_{n}\|+\| x_{n}-z_{n} \| \\
& =\left\|x_{n+1}-x_{n}\right\|+\left\|x_{n}-z_{n}\right\| \rightarrow 0 \text { as } n \rightarrow \infty .
\end{aligned}
$$

Step 3. We show that $\lim _{n \rightarrow \infty}\left\|T z_{n}-z_{n}\right\|=\lim _{n \rightarrow \infty}\left\|y_{n}-z_{n}\right\|=0$. To this end, let $\tilde{x} \in \Omega$. Then we have

$$
\left\|\operatorname{TUT} z_{n}-\tilde{x}\right\|-\left\|z_{n}-\tilde{x}\right\| \mid \leqslant\left\|\operatorname{TUT} z_{n}-z_{n}\right\| .
$$


Taking limit on the both sides and using Step 2, we have

$$
\begin{aligned}
\lim _{n \rightarrow \infty} \mid\left(\| \text { TUT } z_{n}-\tilde{x}\|-\| z_{n}-\tilde{x} \|\right) \mid & =0, \\
\mid \lim _{n \rightarrow \infty}\left(\| \text { TUT } z_{n}-\tilde{x}\|-\| z_{n}-\tilde{x} \|\right) \mid & =0, \\
\lim _{n \rightarrow \infty}\left(\| \text { TUT } z_{n}-\tilde{x}\|-\| z_{n}-\tilde{x} \|\right) & =0 .
\end{aligned}
$$

By nonexpansiveness of TU and T, we get

$$
\left\|\operatorname{TUT} z_{\mathrm{n}}-\tilde{x}\right\| \leqslant\left\|T z_{\mathrm{n}}-\tilde{x}\right\| \leqslant\left\|z_{\mathrm{n}}-\tilde{x}\right\|,
$$

and so,

$$
\left\|\mathrm{TUT} z_{\mathrm{n}}-\tilde{x}\right\|-\left\|z_{\mathrm{n}}-\tilde{x}\right\| \leqslant\left\|\mathrm{T} z_{\mathrm{n}}-\tilde{\mathrm{x}}\right\|-\left\|z_{\mathfrak{n}}-\tilde{x}\right\| \leqslant 0 .
$$

Thus, from (3.21), we induce

$$
\lim _{n \rightarrow \infty}\left(\left\|T z_{n}-\widetilde{x}\right\|-\left\|z_{n}-\widetilde{x}\right\|\right)=0 .
$$

Since $\mathrm{T}$ is firmly nonexpansive and hence strongly nonexpansive [6], we have

$$
\lim _{n \rightarrow \infty}\left\|T z_{n}-z_{n}\right\|=\lim _{n \rightarrow \infty}\left\|y_{n}-z_{n}\right\|=0 .
$$

Step 4. We show that $\limsup _{n \rightarrow \infty}\left\langle\sigma V x^{*}-\mu F x^{*}, T z_{n}-x^{*}\right\rangle \leqslant 0$, where $x^{*}$ is the unique solution of the variational inequality (3.2). Indeed, we can choose a subsequence $\left\{x_{n_{i}}\right\}$ of $\left\{x_{n}\right\}$ such that

$$
\limsup _{n \rightarrow \infty}\left\langle\sigma V x^{*}-\mu F x^{*}, x_{n}-x^{*}\right\rangle=\lim _{i \rightarrow \infty}\left\langle\sigma V x^{*}-\mu F x^{*}, x_{n_{i}}-x^{*}\right\rangle .
$$

Since $\left\{x_{n_{i}}\right\}$ is bounded, there exists a subsequence of $\left\{x_{n_{i}}\right\}$ which converges weakly to a point $\widetilde{x}$. Without loss of generality, we may assume that $\left\{x_{n_{i}}\right\}$ converges weakly to $\widetilde{x}$. Therefore, from Step 2, (3.20), and Lemma 2.4, we have $x_{n_{i}} \rightarrow \widetilde{x} \in \operatorname{Fix}(T U T)$. Since T and $U$ are averaged, by Proposition 2.1 (iv), we have $z \in \operatorname{Fix}(\mathrm{T})$ and $z \in \operatorname{Fix}(\mathrm{U})$, and hence $A z \in \operatorname{Fix}(\mathrm{S})$ by Proposition 2.2 (iii). Thus $z \in \Omega$. Therefore we derive

$$
\limsup _{n \rightarrow \infty}\left\langle\sigma V x^{*}-\mu F x^{*}, x_{n}-x^{*}\right\rangle=\lim _{i \rightarrow \infty}\left\langle\sigma V x^{*}-\mu F x^{*}, x_{n_{i}}-x^{*}\right\rangle=\left\langle\sigma V x^{*}-\mu F x^{*}, \tilde{x}-x^{*}\right\rangle \leqslant 0 .
$$

This together with (3.20) and Step 3 imply that

$$
\limsup _{n \rightarrow \infty}\left\langle\sigma V x^{*}-\mu F x^{*}, T z_{n}-x^{*}\right\rangle \leqslant 0 .
$$

Step 5. We show that $\lim _{n \rightarrow \infty} x_{n}=x^{*}$, where $x^{*}$ is the unique solution of the variational inequality (3.2). We observe that

$$
\left\|\mathrm{T} z_{\mathrm{n}}-x^{*}\right\|^{2}=\left\langle\mathrm{T} z_{\mathrm{n}}-\mathrm{y}_{\mathrm{n}}, \mathrm{T} z_{\mathrm{n}}-x^{*}\right\rangle+\left\langle z_{\mathrm{n}}-x^{*}, \mathrm{~T} z_{\mathrm{n}}-x^{*}\right\rangle .
$$

Since $T$ is a firmly nonexpansive mapping with a fixed point $x^{*}$, we have $\left\langle T z_{n}-z_{n}, T z_{n}-x^{*}\right\rangle \leqslant 0$. Thus we derive

$$
\begin{aligned}
& \left\|\mathrm{T} z_{\mathrm{n}}-x^{*}\right\|^{2} \leqslant\left\langle z_{\mathrm{n}}-x^{*}, \mathrm{~T} z_{\mathrm{n}}-x^{*}\right\rangle \\
& =\left\langle\alpha_{n} \sigma\left(\mathrm{V} x_{n}-\mathrm{V} x^{*}\right)+\left(\mathrm{I}-\alpha_{n} \mu \mathrm{F}\right) x_{n}-\left(\mathrm{I}-\alpha_{n} \mu \mathrm{F}\right) x^{*}, \mathrm{~T} z_{n}-x^{*}\right\rangle+\alpha_{n}\left\langle\sigma \mathrm{V} x^{*}-\mu \mathrm{F} x^{*}, \mathrm{~T} z_{n}-x^{*}\right\rangle \\
& \leqslant\left(\alpha_{n} \sigma l\left\|x_{n}-x^{*}\right\|+\left(1-\alpha_{n} \tau\right)\left\|x_{n}-x^{*}\right\|\right)\left\|T z_{n}-x^{*}\right\|+\alpha_{n}\left\langle\sigma V x^{*}-\mu F x^{*}, T z_{n}-x^{*}\right\rangle \\
& =\left(1-\alpha_{n}(\tau-\sigma l)\right)\left\|x_{n}-x^{*}\right\|\left\|T z_{n}-x^{*}\right\|+\alpha_{n}\left\langle\sigma V x^{*}-\mu F x^{*}, T z_{n}-x^{*}\right\rangle \\
& \leqslant \frac{1-\alpha_{n}(\tau-\sigma l)}{2}\left\|x_{n}-x^{*}\right\|^{2}+\frac{1}{2}\left\|T z_{n}-x^{*}\right\|^{2}+\alpha_{n}\left\langle\sigma V x^{*}-\mu F x^{*}, T z_{n}-x^{*}\right\rangle \text {. }
\end{aligned}
$$

It follows that

$$
\left\|\mathrm{T} z_{n}-x^{*}\right\|^{2} \leqslant\left[1-\alpha_{n}(\tau-\sigma l)\right]\left\|x_{n}-x^{*}\right\|^{2}+2 \alpha_{n}\left\langle\sigma V x^{*}-\mu F x^{*}, T z_{n}-x^{*}\right\rangle .
$$


From (3.13) and (3.22), we have

$$
\begin{aligned}
\left\|x_{n+1}-x^{*}\right\|^{2} & =\left\|T U T z_{n}-x^{*}\right\|^{2} \\
& \leqslant\left\|T z_{n}-x^{*}\right\|^{2} \\
& \leqslant\left[1-\alpha_{n}(\tau-\sigma l)\right]\left\|x_{n}-x^{*}\right\|^{2}+\alpha_{n}(\tau-\sigma l) \frac{2}{\tau-\sigma l}\left\langle\sigma V x^{*}-\mu F x^{*}, T z_{n}-x^{*}\right\rangle .
\end{aligned}
$$

Put $\lambda_{n}=\alpha_{n}(\tau-\sigma l)$ and

$$
\delta_{n}=\frac{2}{\tau-\sigma l}\left\langle\sigma V x^{*}-\mu F x^{*}, T z_{n}-x^{*}\right\rangle
$$

It can be easily seen from Step 4 and conditions (C1) and (C2) that $\lambda_{n} \rightarrow 0, \sum_{n=0}^{\infty} \lambda_{n}=\infty$, and $\limsup _{n \rightarrow \infty} \delta_{n} \leqslant 0$. Since (3.23) reduces to

$$
\left\|x_{n+1}-x^{*}\right\|^{2} \leqslant\left(1-\lambda_{n}\right)\left\|x_{n}-x^{*}\right\|^{2}+\lambda_{n} \delta_{n}
$$

by Lemma 2.7, we conclude that $\lim _{n \rightarrow \infty}\left\|x_{n}-x^{*}\right\|=0$. This completes the proof.

Putting $\mu=1$ and $F=I$ in Theorem 3.5, we obtain the following corollary.

Corollary 3.6. Let $\left\{x_{n}\right\}$ be generated by the following algorithm:

$$
x_{n+1}=T\left[I-\gamma A^{*}(I-S) A\right] T\left[\alpha_{n} \sigma V x_{n}+\left(1-\alpha_{n}\right) x_{n}\right], \quad n \geqslant 0 .
$$

Assume that the sequence $\left\{\alpha_{n}\right\} \in[0,1]$ satisfies the conditions $(\mathrm{C} 1)$ and $(\mathrm{C} 2)$ in Theorem 3.5. Then $\left\{x_{n}\right\}$ converges strongly to a point $x^{*} \in \Omega$ which is the unique solution of the variational inequality (3.9).

Putting $\mathrm{V}=0$ in (3.24), we get the following corollary.

Corollary 3.7. Let $\left\{x_{n}\right\}$ be generated by the following algorithm:

$$
x_{n+1}=T\left[I-\gamma A^{*}(I-S) A\right] T\left[\left(1-\alpha_{n}\right) x_{n}\right], \quad n \geqslant 0 .
$$

Assume that the sequence $\left\{\alpha_{n}\right\}$ satisfies the conditions (C1) and (C2) in Theorem 3.5. Then $\left\{x_{n}\right\}$ converges strongly to a point $x^{*}$ which is the minimum norm solution of the SCFPP (1.2).

Remark 3.8.

1) It is well-known that the metric projection is firmly nonexpansive and hence nonexpansive. Thus iterative algorithms (3.1) and (3.13) are more general than [16, iterative algorithms (3.1) and (3.11)], respectively. Indeed, if we consider $T=P_{C}, S=P_{Q}, \operatorname{Fix}(T)=C$ and $\operatorname{Fix}(S)=Q$, then Theorem 3.2 and Theorem 3.5 generalize [16, Theorem 3.2] and [16, Theorem 3.5], respectively.

2) Theorem 3.2 and Theorem 3.5 also improve [2, Theorem 3.5 and Theorem 3.7] and [31, Theorem 3.1 and Theorem 3.5], respectively.

3) Corollary 3.3 and Corollary 3.6 generalize [16, Corollary 3.3 and Corollary 3.6] and [31, Corollary 3.2 and Corollary 3.7], respectively.

4) Corollary 3.4 and Corollary 3.7 improve [31, Corollary 3.3 and Corollary 3.9], respectively.

\section{Applications}

Now, as in [2], we apply our iterative algorithms to study some problems from nonlinear and convex analysis. 


\subsection{Variational problems via resolvent operators}

For a given a maximal monotone operator $\mathrm{M}: \mathrm{H}_{1} \rightarrow 2^{\mathrm{H}_{1}}$, it is well-known that its associated resolvent operator $J_{r}^{M}=(I+r M)^{-1}$ is firmly nonexpansive and $0 \in M(x) \Longleftrightarrow J_{r}^{M}(x)=x$ for $r>0$; see, for instance, $[1,24]$. This means zeros of $M$ are exactly fixed points of its resolvent operator. Let $T=J_{r}^{M}$ and $S=J_{r}^{N}$, where $\mathrm{N}: \mathrm{H}_{2} \rightarrow 2^{\mathrm{H}_{2}}$ is a maximal monotone operator. We consider the problem of finding $x^{*} \in \Omega_{1}$ such that

$$
\left\langle\sigma \vee x^{*}-\mu F x^{*}, \tilde{x}-x^{*}\right\rangle \leqslant 0, \quad \forall \tilde{x} \in \Omega_{1},
$$

where $\Omega_{1}=M^{-1}(0) \cap A^{-1}\left(N^{-1}(0)\right)$. Under these restrictions, iterative algorithms (3.1) and (3.13) reduce the following iterative algorithms, respectively.

Algorithm 4.1. For any $t \in\left(0, \frac{1}{\tau-\sigma l}\right)$, define a net $\left\{x_{t}\right\} \subset H_{1}$ in an implicit way:

$$
x_{t}=J_{r}^{M}\left[I-\gamma A^{*}\left(I-J_{r}^{N}\right) A\right] J_{r}^{M}\left[t \sigma V x_{t}+(I-t \mu F) x_{t}\right] .
$$

Algorithm 4.2. For an arbitrarily chosen $x_{0} \in \mathrm{H}_{1}$, compute in an explicit way:

$$
x_{n+1}=J_{r}^{M}\left[I-\gamma A^{*}\left(I-J_{r}^{N}\right) A\right] J_{r}^{M}\left[\alpha_{n} \sigma V x_{n}+\left(I-\alpha_{n} \mu F\right) x_{n}\right], \quad n \geqslant 0,
$$

where $\left\{\alpha_{n}\right\} \subset[0,1]$.

Since the resolvent operators are firmly nonexpansive, the strong convergence of the net $\left\{x_{t}\right\}$ (respectively, the sequence $\left\{x_{n}\right\}$ ) generated by Algorithm 4.1 (respectively, Algorithm 4.2) can be derived from Theorem 3.2 (respectively, Theorem 3.5).

\subsection{Equilibrium problems via resolvent operators}

Let $C$ be a nonempty closed convex subset of a Hilbert space, and let $\Theta: C \times C \rightarrow \mathbb{R}$ be a bifunction. Consider the following equilibrium problem: find $z \in \mathrm{C}$ such that

$$
\Theta(z, y) \geqslant 0, \quad \forall y \in C .
$$

The set of all $z \in C$ which satisfies (4.1) is denoted by $\operatorname{EP}(C, \Theta)$, i.e.,

$$
E F(C, \Theta)=\{z \in C: \Theta(z, y) \geqslant 0, \forall y \in C\}
$$

For solving the equilibrium problem, let us assume that the bifunction $\Theta$ satisfies the following conditions:

(H1) $\Theta(x, x)=0, \forall x-n C$;

(H2) $\Theta$ is monotone, i.e., $\Theta(x, y)+\Theta(y, x) \leqslant 0, \forall x, y \in C$;

(H3) $\lim _{\mathrm{t} \downarrow 0} \Theta(t z+(1-t) x, y) \leqslant \Theta(x, y), \forall x, y, z \in C$;

(H4) for each $x \in H, y \mapsto \Theta(x, y)$ is convex and lower semicontinuous.

It is well-known $([3,12])$ that the associated resolvent operator $\mathrm{T}_{\mathrm{r}}^{\Theta}: \mathrm{H} \rightarrow \mathrm{C}$ defined by

$$
\mathrm{T}_{\mathrm{r}}^{\Theta} x=\left\{z \in \mathrm{C}: \Theta(z, y)+\frac{1}{\mathrm{r}}\langle y-z, z-x\rangle \geqslant 0, \forall y \in C\right\},
$$

is firmly nonexpansive and $\operatorname{Fix}\left(T_{r}^{\Theta}\right)=E P(C, \Theta)$. Let $T=T_{r}^{\Theta}$ and $S=S_{v}^{\Phi}$, where $\Phi: Q \times Q \rightarrow \mathbb{R}$ is another function. We consider the problem of finding $x^{*} \in \Omega_{2}$ such that

$$
\left\langle\sigma \vee x^{*}-\mu F x^{*}, \widetilde{x}-x^{*}\right\rangle \leqslant 0, \quad \forall \widetilde{x} \in \Omega_{2},
$$

where $\Omega_{2}=\operatorname{EP}(C, \Theta) \cap A^{-1}(E P(Q, \Phi))$. Under these restrictions, iterative algorithms (3.1) and (3.13) reduces the following iterative algorithms, respectively. 
Algorithm 4.3. For any $t \in\left(0, \frac{1}{\tau-\sigma l}\right)$, define a net $\left\{x_{\mathrm{t}}\right\} \subset \mathrm{H}_{1}$ in an implicit way:

$$
x_{t}=T_{r}^{\Theta}\left[I-\gamma A^{*}\left(I-S_{v}^{\Phi}\right) A\right] T_{r}^{\Theta}\left[t \sigma V x_{t}+(I-t \mu F) x_{t}\right] .
$$

Algorithm 4.4. For an arbitrarily chosen $x_{0} \in \mathrm{H}_{1}$, compute in an explicit way:

$$
x_{n+1}=T_{r}^{\Theta}\left[I-\gamma A^{*}\left(I-S_{v}^{\Phi}\right) A\right] T_{r}^{\Theta}\left[\alpha_{n} \sigma V x_{n}+\left(I-\alpha_{n} \mu F\right) x_{n}\right], \quad n \geqslant 0,
$$

where $\left\{\alpha_{n}\right\} \subset[0,1]$.

Since the resolvent operators are firmly nonexpansive, the strong convergence of the net $\left\{x_{t}\right\}$ (respectively, the sequence $\left\{x_{n}\right\}$ ) generated by Algorithm 4.3 (respectively, Algorithm 4.4) can be derived from Theorem 3.2 (respectively, Theorem 3.5).

\section{Acknowledgment}

This study was supported by research funds from Dong-A University.

The author would like to thank the anonymous reviewers for their careful reading and valuable suggests, which improved the presentation of this manuscript.

\section{References}

[1] R. P. Agarwal, D. O’Regan, D. R. Sahu, Fixed point theory for Lipschitzian-type mappings with applications, Topological Fixed Point Theory and Its Applications, Springer, New York, (2009). 2, 2.3, 4.1

[2] Q. H. Ansari, A. Rehan, C.-F. Wen, Implicit and explicit algorithms for split common fixed point problems, J. Nonlinear Convex Anal., 17 (2016), 1281-1397. 1, 2, 3.8, 4

[3] E. Blum, W. Oettli, From optimization and variational inequalities to equilibrium problems, Math. Student, 63 (1994), 123-146. 4.2

[4] C. Byrne, Iterative oblique projection onto convex sets and the split feasibility problem, Inverse Problems, 18 (2002), 441-453. 1

[5] C. Byrne, A unified treatment of some iterative algorithms in signal processing and image reconstruction, Inverse Problems, 20 (2004), 103-120. 2, 2.1

[6] A. Cegielski, Iterative methods for fixed point problems in Hilbert spaces, Lecture Notes in Mathematics, Springer, Heidelberg, (2012). 2, 3

[7] Y. Censor, T. Bortfeld, B. Martin, A. Tronfimov, A unified approach for inversion problems in intensity-modulated radiation therapy, Phys. Med. Biol., 51 (2006), 2353-2365. 1

[8] Y. Censor, T. Elfving, A multiprojection algorithm using Bregman projections in a product space, Numer. Algorithms, 8 (1994), 221-239. 1

[9] Y. Censor, T. Elfving, N. Kopf, T. Bortfeld, The multiple-sets split feasibility problem and its applications for inverse problems, Inverse Problems, 21 (2005), 2071-2084. 1

[10] Y. Censor, A. Motova, A. Segal, Perturbed projections and subgradient projections for the multiple-sets split feasibility problem, J. Math. Anal. Appl., 327 (2007), 1244-1256. 1

[11] Y. Censor, A. Segal, The split common fixed point problem for directed operators, J. Convex Anal., 16 (2009), 587-600. 1

[12] P. L. Combettes, S. A. Hirstoaga, Equilibrium programming in Hilbert spaces, J. Nonlinear Convex Anal., 6 (2005), 117-136. 4.2

[13] H.-H. Cui, F.-H. Wang, Iterative methods for the split common fixed point problem in Hilbert spaces, Fixed Point Theory Appl., 2014 (2014), 8 pages. 1

[14] Y.-Z. Dang, Y. Gao, The strong convergence of a KM-CQ-like algorithm for a split feasibility problem, Inverse Problems, 27 (2011), 9 pages. 1

[15] K. Goebel, W. A. Kirk, Topics in metric fixed point theory, Cambridge Studies in Advanced Mathematics, Cambridge University Press, Cambridge, (1990). 2.4

[16] J. S. Jung, Iterative algorithms based on the hybrid steepest descent method for the split feasibility problem, J. Nonlinear Sci. Appl., 9 (2016), 4214-4225. 1, 1, 3.8

[17] R. Kraikaew, S. Saejung, On split common fixed point problems, J. Math. Anal. Appl., 415 (2014), 513-524. 1

[18] A. Latif, D. Y. Sahu, Q. H. Ansari, Variable KM-like algorithms for fixed point problems and split feasibility problems, Fixed Point Theory Appl., 2014 (2014), 20 pages. 1

[19] G. J. Minty, On the generalization of a direct method of the calculus of variations, Bull. Amer. Math. Soc., 73 (1967), 315-321. 2.5 
[20] A. Moudafi, The split common fixed-point problem for demicontractive mappings, Inverse Problems, 26 (2010), 6 pages. 1

[21] A. Moudafi, A note on the split common fixed-point problem for quasi-nonexpansive operators, Nonlinear Anal., 74 (2011), 4083-4087. 1

[22] B. Qu, N.-H. Xiu, A note on the CQ algorithm for the split feasibility problem, Inverse Problems, 21 (2005), $1655-1665$. 1

[23] T. Suzuki, Strong convergence of Krasnoselskii and Mann's type sequences for one-parameter nonexpansive semigroups without Bochner integrals, J. Math. Anal. Appl., 305 (2005), 227-239. 2.6

[24] W. Takahashi, Nonlinear functional analysis, Fixed point theory and its applications, Yokohama Publishers, Yokohama, (2000). 4.1

[25] F.-H. Wang, H.-K. Xu, Approximating curve and strong convergence of the CQ algorithm for the split feasibility problem, J. Inequal. Appl., 2010 (2010), 13 pages. 1

[26] H.-K. Xu, Iterative algorithms for nonlinear operators, J. London Math. Soc., 66 (2002), 240-256. 2.7

[27] H.-K. Xu, Iterative methods for the split feasibility problem in infinite-dimensional Hilbert spaces, Inverse Problems, 26 (2010), 17 pages. 1

[28] I. Yamada, The hybrid steepest descent method for the variational inequality problem over the intersection of fixed point sets of nonexpansive mappings, Inherently parallel algorithms in feasibility and optimization and their applications, Haifa, (2000), Stud. Comput. Math., North-Holland, Amsterdam, 8 (2001), 473-504. 1, 1, 2

[29] I. Yamada, N. Ogura, Hybrid steepest descent method for variational inequality problem over the fixed point set of certain quasi-nonexpansive mappings, Numer. Funct. Anal. Optim., 25 (2004), 619-655. 2

[30] Q.-Z. Yang, The relaxed CQ algorithm solving the split feasibility problem, Inverse Problems, 20 (2004), 1261-1266. 1

[31] Y.-H. Yao, P.-X. Yang, S. M. Kang, Composite projection algorithms for the split feasibility problem, Math. Comput. Modelling, 57 (2013), 693-700. 3.8

[32] J.-L. Zhao, Q.-Z. Yang, Several solution methods for the split feasibility problem, Inverse Problems, 21 (2005), 1791-1799. 1 